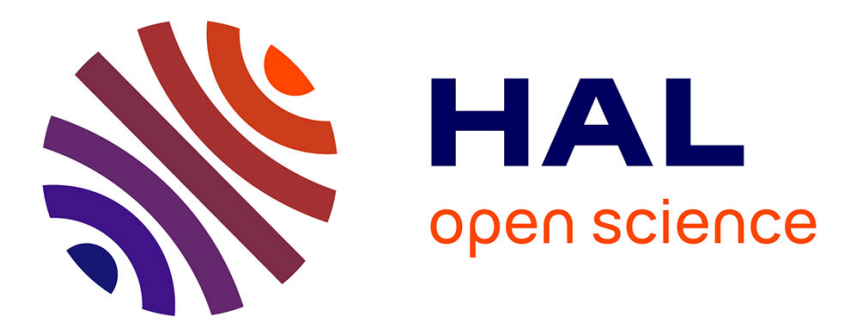

\title{
A theoretical model for the electrical properties of chromium thin films sputter deposited at oblique incidence
}

\author{
Aurélien Besnard, Nicolas Martin, Luc Carpentier, Bruno Gallas
}

\section{To cite this version:}

Aurélien Besnard, Nicolas Martin, Luc Carpentier, Bruno Gallas. A theoretical model for the electrical properties of chromium thin films sputter deposited at oblique incidence. Journal of Physics D: Applied Physics, 2011, 44 (21), pp.215301. 10.1088/0022-3727/44/21/215301 . hal-00618210

\author{
HAL Id: hal-00618210 \\ https://hal.science/hal-00618210
}

Submitted on 1 Sep 2011

HAL is a multi-disciplinary open access archive for the deposit and dissemination of scientific research documents, whether they are published or not. The documents may come from teaching and research institutions in France or abroad, or from public or private research centers.
L'archive ouverte pluridisciplinaire HAL, est destinée au dépôt et à la diffusion de documents scientifiques de niveau recherche, publiés ou non, émanant des établissements d'enseignement et de recherche français ou étrangers, des laboratoires publics ou privés. 


\title{
A theoretical model for the electrical properties of chromium thin films sputter deposited at oblique incidence
}

\author{
Aurélien BESNARD ${ }^{1}$, Nicolas MARTIN ${ }^{1, *}$, Luc CARPENTIER $^{1}$, Bruno GALLAS $^{2}$ \\ ${ }^{1}$ Institut FEMTO-ST, Université de Franche-Comté, CNRS, ENSMM, UTBM \\ 32, Avenue de l'Observatoire 25044 BESANCON Cedex, France \\ ${ }^{2}$ Institut des NanoSciences de Paris, CNRS, Université Pierre et Marie Curie \\ 4, Place Jussieu, boîte courrier 840, 75252 Paris Cedex 05, France
}

Chromium thin films are prepared by magnetron sputtering using the GLancing Angle Deposition (GLAD) method. The electrical conductivity of the films exhibiting an inclined columnar structure is investigated. An analytical three dimensional model is proposed in order to predict the evolution of the electrical conductivity as a function of the columns orientation. This model is based on two types of electron scattering mechanisms, which are simultaneously operational: the isotropic background scattering at the grain boundary and the scattering due to electron dispersion at the column boundary. The developed analytical expressions enable systematic studies of different process and film parameters, especially the column angle $\beta$ as well as the sputtering pressure used during the GLAD deposition. The theoretical and experimental results are compared. It is found that the column angle $\beta$ must reach a threshold value to significantly reduce the electrical conductivity of chromium thin films close to one order of magnitude. The effect of the sputtering pressure on the films 
structure and their electrical behaviours are also studied and well predicted by the proposed model. A comprehensive discussion of the applicability of the model is finally presented.

Keywords: GLAD, chromium films, modelling, conductivity, inclined columns.

*Corresponding author: nicolas.martin@femto-st.ft; Tel.: +33 3818539 69; Fax: +33 381 853998 


\section{Introduction}

The electrical properties of thin polycrystalline films strongly deviate from the corresponding bulk-single crystalline solid. It is commonly observed that the conductivity of films is reduced mainly due to a decrease of the carriers mean free path [1]. For metallic compounds, the electrical conductivity is closely linked to the electron behaviours. Several mechanisms have to be considered in order to understand the electronic transport phenomena in thin solid films. Electron scattering at the grain boundary prevails and it has to be analyzed taking into account, the crystallite grain size, the direction of grain boundaries compared to the applied electric field (perpendicular or parallel), the thickness of the film, etc. Thus, the electrical properties of metallic polycrystalline materials become even more complex for thin films exhibiting some anisotropic structures such as an oriented columnar architecture [2]. To this aim, various theoretical models were developed to predict the evolution of metallic films conductivity as a function of their microstructure, especially the correlation with the grain size and the thickness of the films [3-8]. Furthermore, very little is known about the conductivity changes due to a controlled orientation of the columnar structure in thin films [9]. It is even more verified for theoretical models taking into account these special architectures and being able to calculate the transport properties in such anisotropic materials [10].

The purpose of this paper is to investigate the electrical conductivity of metallic thin films exhibiting an inclined columnar structure. Chromium thin films were sputter deposited implementing the GLancing Angle Deposition (GLAD) method [11, 12]. Three sputtering pressures were used and the incident angle $\alpha$ of the sputtered particles was systematically changed from 0 to $80^{\circ}$. Oriented chromium columns were produced with various column angles $\beta$ closely linked to the sputtering pressure and incident angle $\alpha$. At low sputtering pressure (e.g. $0.11 \mathrm{~Pa}$ ), the mean free path of sputtered particles is large enough to get a ballistic trajectory of the particles impinging on the growing film. On the other hand, a high 
sputtering pressure (e.g. $0.53 \mathrm{~Pa}$ ) favours scattering of the sputtered particles and reduces the columnar inclination. As a result, sputtering pressure as well as incident angle $\alpha$ showed a significant influence on the electrical conductivity of the films. Similarly, a theoretical model is proposed and detailed calculations of the electrical transport properties are compared with experimental results.

\section{Theory}

In polycrystalline thin films prepared by conventional sputtering, the first zone of the structural zone model proposed by Thornton qualitatively predicts a fine and dense columnar microstructure [13]. Grains are not isotropic in form and the columnar shape (with the column axis normal to the film plane) is commonly met in thin films deposited by evaporation or sputtering. Since such columns generally extend from the substrate to the surface of the film, boundaries and voids between columns have to be taken into account first. In addition, if the crystallite grain size is in the same range of values as the bulk electron mean free path, the column boundaries are the most significant in electron scattering. As a result, it is assumed that the effect of column scattering is dominant whereas the contribution of the grain boundary scattering becomes negligible in the conduction mechanism and is merged in the background scattering. Thus, the Mayadas and Schatzkes model [14] using column-like grain can be extended to polycrystalline columnar structures. Such a model only considers the effect due to the planes perpendicular to the applied electric field. For our oriented thin films composed of tilted columns, the three-dimensional model proposed by Pichard et al. [15] appears as the most satisfying theory. In fact, in their model, boundaries are represented by three arrays of planar potentials perpendicular to the $\mathrm{x}, \mathrm{y}$ and $\mathrm{z}$ axes, respectively (Fig. 1a, $\mathrm{b}$ and c). We improved this approach assuming that all columns are inclined with the column angle $\beta\left({ }^{\circ}\right)$ in the $(\mathrm{x}, \mathrm{z})$ plane. The cross-section of each column is assumed as a square with a 
side $D_{x}=D_{y}=D$ and height $D_{z}=d$ (Fig. 2), where $d$ is the film thickness (m). Using the polar coordinates $(r, \varphi, \theta)$ defined in figure 1 , the distances $\mathrm{L}_{\mathrm{x}}, \mathrm{L}_{\mathrm{y}}$ and $\mathrm{L}_{\mathrm{z}}(\mathrm{m})$ measured between two successive points of the electron trajectory located on the planar boundaries distributed perpendicular to the $\mathrm{x}, \mathrm{y}$ and $\mathrm{z}$ axes (Fig. 1) are expressed as:

$$
\begin{aligned}
& L_{x}=D \cdot \cos ^{-1} \beta \cdot|\cos \varphi|^{-1} \cdot|\sin \theta|^{-1} \\
& L_{y}=D \cdot|\sin \varphi|^{-1} \cdot|\sin \theta|^{-1} \\
& L_{z}=d \cdot|\cos \theta|^{-1}
\end{aligned}
$$

For $\varphi$ and $\theta$ direction angles, Pichard et al. [15] assumed as "t", the fraction of electrons transmitted through any planar potential. The total probability "P" that an electron travels a path of length "L" without being scattered may be written as:

$$
P=e^{-\frac{L}{\lambda}}
$$

where $\lambda$ is the mean free path of the electrons related to the boundary effect $(\mathrm{m})$, or:

$$
P=t^{\frac{L}{L x}+\frac{L}{L y}+\frac{L}{L z}}
$$

The transmission coefficient " $\mathrm{t}$ " through a potential is related to the density of the material “ $\xi "$ according to the following relationships:

$$
t=t_{0} e^{-A\left(\xi-\xi_{0}\right)}
$$

where " $\mathrm{t}_{0}$ " is related to the electrons flux impinging on the boundary (planar potential), "A" is a constant depending on the thickness and absorption of the barrier potential (i.e. cross-section of electron absorption by the material), and " $\xi_{0}$ " is the density of the bulk material $\left(\mathrm{kg} \cdot \mathrm{m}^{-3}\right)$. Taking into account previous results published by Paik et al. [16], density " $\xi$ ” of oriented thin films produced by GLAD, can be related to the incident angle $\alpha$ of the particles flux by:

$$
\xi=\frac{\xi_{\alpha=0^{\circ}}}{1+c \tan (\alpha)}
$$


where $\xi_{\alpha=0^{\circ}}$ is the density of the film deposited at an incident angle $\alpha=0^{\circ}\left(\mathrm{kg} \cdot \mathrm{m}^{-3}\right)$ and "c" is a constant, which is proportional to the ratio of the shadowing step height to the column thickness. At first, we suppose that the density of the bulk material is the same as the density of the film deposited at an incident angle $\alpha=0^{\circ}$ (i.e. $\xi_{0}=\xi_{\alpha=0^{\circ}}$ ).

\section{Equation (7) becomes:}

$t=t_{0} e^{\frac{A \xi_{0} c \tan (\alpha)}{1+c \tan (\alpha)}}$

From equations (4) and (5) and introducing equations (1)-(3) in (5), the mean free path of electrons " $\lambda_{c}{ }^{-1}$ " in a column is given by:

$\lambda_{c}^{-1}=\ln \frac{1}{t} \cdot\left(D^{-1} \cdot \cos \beta \cdot|\cos \varphi| \cdot|\sin \theta|+D^{-1} \cdot|\sin \varphi| \cdot|\sin \theta|+d^{-1} \cdot|\cos \theta|\right)$

and then:

$\lambda_{c}^{-1}=\ln \frac{1}{t} \cdot D^{-1} \cdot\left((\cos \beta \cdot|\cos \varphi|+|\sin \varphi|) \cdot|\sin \theta|+D \cdot d^{-1} \cdot|\cos \theta|\right)$

One can define " $\mathrm{C}(\beta)$ ") as:

$$
C(\beta)=\cos \beta \cdot|\cos \varphi|+|\sin \varphi|
$$

For $\beta \in[0, \pi / 2]$ and $\varphi \in[0,2 \pi]$, the average value of $\mathrm{C}(\beta)$ is:

$$
C(\beta)=\frac{1}{2 \pi} \int_{0}^{2 \pi}(\cos \beta \cdot|\cos \varphi|+|\sin \varphi|) d \varphi=\frac{2}{\pi}(1+\cos (\beta))
$$

The $\varphi$ angle can take any value in the range $[0,2 \pi]$. So $C(\beta)$ can be replaced in equation (10) and the mean free path becomes:

$$
\lambda_{c}^{-1}=\ln \frac{1}{t} \cdot D^{-1} \cdot\left(C(\beta) \cdot|\sin \theta|+D \cdot d^{-1} \cdot|\cos \theta|\right)
$$

Since:

$$
C(\beta=0)=|\cos \varphi|+|\sin \varphi| \quad \frac{4}{\pi}
$$


and introducing in (13), it gives:

$$
\lambda_{c}^{-1}=\ln \frac{1}{t} \cdot D^{-1} \cdot\left(C(\beta) \cdot C(0)+\left(D \cdot d^{-1}-C(\beta)\right) \cdot|\cos \theta|\right)
$$

Two electrons scattering mechanisms are only assumed, i.e. the isotropic background scattering defined by " $\lambda_{0}$ " and the column boundary scattering defined by " $\lambda_{\mathrm{c}}$ ".

The global mean free path " $\lambda$ ” is given by the Matthiessen's rule:

$$
\lambda^{-1}=\lambda_{0}^{-1}+\lambda_{c}^{-1}
$$

Hence:

$$
\lambda^{-1}=\lambda_{0}^{-1}+\ln \frac{1}{t} \cdot D^{-1} \cdot\left(C(\beta) \cdot C(0)+\left(D \cdot d^{-1}-C(\beta)\right) \cdot|\cos \theta|\right)
$$

Here we define for convenience a grain parameter " $v(\beta)$ " as:

$$
\nu(\beta)=D \cdot \lambda_{0}^{-1} \cdot \ln \left(\frac{1}{t}\right)^{-1}
$$

or

$$
\nu(\beta)=D \cdot \lambda_{0}^{-1} \cdot\left(\ln \left(\frac{1}{t_{0}}\right)-\frac{A \cdot \xi_{0} \cdot c \cdot \tan (\alpha)}{1+c \cdot \tan (\alpha)}\right)^{-1}
$$

The transmission coefficient $t$ is assumed to vary with the column angle $\beta$.

So:

$$
\lambda_{0} \cdot \lambda^{-1}=1+\frac{C(\beta) \cdot C(0)}{v}+\frac{D \cdot d^{-1}-C(\beta)}{v} \cdot|\cos \theta|
$$

Using the Boltzmann equation, when an electric field is applied according to the " $\mathrm{x}$ " direction, the film conductivity is found to be $[10,17]$

$$
\sigma=\frac{3 \cdot \sigma_{0}}{4 \pi} \int_{0}^{2 \pi} \int_{0}^{\pi} \frac{\cos ^{2} \phi \cdot \sin ^{3} \varphi}{\lambda_{0} \cdot \lambda^{-1}} d \theta d \varphi
$$

where $\sigma_{0}$ is the electrical conductivity of the bulk material $\left(\mathrm{S} \mathrm{m}^{-1}\right)$, 
and thus:

$$
\frac{\sigma}{\sigma_{0}}=\frac{3}{4} \int_{0}^{\pi} \frac{\sin ^{3} \theta}{1+\frac{C(\beta) \cdot C(0)}{v}+\frac{D \cdot d^{-1}-C(\beta)}{v} \cdot|\cos \theta|} d \theta
$$

Because of the symmetry of the function related to $\pi / 2$, equation (22) becomes:

$$
\frac{\sigma}{\sigma_{0}}=\frac{3}{2} \int_{0}^{\pi / 2} \frac{\sin ^{3} \theta}{1+\frac{C(\beta) \cdot C(0)}{v}+\frac{D \cdot d^{-1}-C(\beta)}{v} \cdot \cos \theta} d \theta
$$

Assuming $\mathrm{u}=\cos \theta, \mathrm{du}=-\sin \theta \mathrm{d} \theta$

$$
\frac{\sigma}{\sigma_{0}}=\frac{3}{2} \int_{0}^{1} \frac{1-u^{2}}{1+\frac{C(\beta) \cdot C(0)}{v}+\frac{D \cdot d^{-1}-C(\beta)}{v} \cdot u} d u
$$

and after integration, the electrical conductivity ratio or the electrical resistivity ratio is finally expressed by:

$$
\frac{\sigma}{\sigma_{0}}=\frac{\rho_{0}}{\rho}=\left[\frac{3}{2} \cdot \frac{v(\alpha)}{D \cdot d^{-1}-C(\beta)}\left[\begin{array}{l}
\left(1-\left(\frac{v(\alpha)+C(\beta) \cdot C(0)}{D \cdot d^{-1}-C(\beta)}\right)^{2}\right) \times \\
\ln \left(1+\frac{D \cdot d^{-1}-C(\beta)}{v(\alpha)+C(\beta) \cdot C(0)}\right)+\frac{v(\alpha)+C(\beta) \cdot C(0)}{D \cdot d^{-1}-C(\beta)}-\frac{1}{2}
\end{array}\right]\right]
$$

with " $\rho$ " is the electrical resistivity of the GLAD film $(\Omega \mathrm{m})$ and " $\rho_{0}$ " the electrical resistivity of the bulk material $(\Omega \mathrm{m})$.

\section{Experimental details}

Chromium thin films were deposited on glass and (100) silicon substrates by DC magnetron sputtering from a chromium metallic target (purity $99.7 \%$ ). The metallic target was sputtered with a constant current density $\mathrm{J}_{\mathrm{Cr}}=200$ A.m ${ }^{-2}$ in argon atmosphere. The substrates were grounded and kept at room temperature. Argon mass flow rate was set 
constant in order to reach a sputtering pressure of $0.11,0.40$ or $0.53 \mathrm{~Pa}$ (pumping speed was maintained at $S=10 \mathrm{~L} . \mathrm{s}^{-1}$ ). The deposition time was adjusted in order to deposit a constant thickness close to $1 \mu \mathrm{m}$. This latter was checked after deposition by profilometry. The homemade GLAD substrate holder allowed an orientation change of the incident angle of the particles flux $\alpha$ from 0 to $90^{\circ}$. This incident angle was taken between the normal of the substrate and the normal of the target (i.e. the main direction of the sputtered atoms flux). The crystallographic structure was investigated by X-ray diffraction (XRD) using monochromatic Co $\mathrm{K}_{\alpha}$ radiation with a Bragg-Brentano configuration $\theta / 2 \theta$. The Debye-Scherrer method was used to calculate the crystallite grain size. Column angle $\beta$ was measured from scanning electron microscopy (SEM) observations on the fractured cross-section of the films deposited on silicon substrates. The DC electrical conductivity of the films deposited on glass substrates was measured at $300 \mathrm{~K}$ in the Van Der Pauw geometry [18].

\section{Results and discussion}

In agreement with the structural zone model proposed by Thornton [13], observation by SEM of the cross-section of the films deposited with a perpendicular incidence of the particles flux $\left(\alpha=0^{\circ}\right)$ exhibits a typical columnar microstructure. As expected, this kind of cross-section morphology corresponds to the first zone of the Thornton's model since the sputtering pressure was $0.11,0.40$ and $0.53 \mathrm{~Pa}$ and the substrate temperature was a few tens of the chromium melting point $(2173 \mathrm{~K})$. Columns consist of inverted cone-like units capped by domes. Films produced in this first zone appear as a quite dense structure with a column width close to $100 \mathrm{~nm}$. They are more or less separated by voided boundaries that are few nanometres wide. A similar columnar structure was produced for films prepared at sputtering pressures of $0.11,0.40$ and $0.53 \mathrm{~Pa}$ (Fig. 3). Increasing the incident angle $\alpha$ of the particles flux, oriented columns appear with similar features observed for films deposited with a 
normal incidence. However, such inclined columns become

separated and more visible. A much more porous and fibrous structure can be distinguished as the incident angle $\alpha$ increases and the sputtering pressure reduces down to $0.11 \mathrm{~Pa}$ (Fig. 3). It is also worth of noticing that the column angle $\beta$ is influenced by the sputtering pressure. As the incident angle $\alpha$ tends to $90^{\circ}$, the column angle $\beta$ asymptotically reaches $24^{\circ}$ for $0.53 \mathrm{~Pa}$ whereas $\beta$ angle tends to $35^{\circ}$ for $0.40 \mathrm{~Pa}$ and $\beta$ tends to $60^{\circ}$ for $0.11 \mathrm{~Pa}$ (Fig. 4). Thus, for both sputtering pressures, $\beta$ versus $\alpha$ well follows the empirical tangent rule $(\tan \alpha=2 \tan \beta)$ up to an incident angle $\alpha$ close to $60^{\circ}$, as commonly observed for several compounds [19]. A good agreement with Tait's model [20] is especially measured for the lowest sputtering pressure. Afterwards, a clear discrepancy is measured, which is even more significant for a high sputtering pressure (e.g. $0.53 \mathrm{~Pa}$ ). A saturation of the column angle $\beta$ occurs. It is mainly attributed to the mean free path of the sputtered particles and the sputtering emission (the angular distribution of sputtered atoms follows an under-cosine law for ions energy lower than $1 \mathrm{keV}$ [21]) peculiar to the target material. Due to enhanced collisions between sputtered particles and argon atoms, flux of chromium atoms impinging on the growing film becomes less directional [22]. The angular distribution of sputtered chromium incoming from the target surface is spread leading to a more randomized flux rather than pure ballistic and consequently, a saturation of the column angle $\beta$. Therefore, sputtering pressure and incident angle $\alpha$ both influence the final microstructure of the deposited films. Incident angle $\alpha$ affects the orientation of the columns according to empirical laws like tangent rules or geometric analyses suggested by Tail et al. [20]. These laws are especially valuable for evaporation methods since the ballistic character of particles impinging on the growing film are acceptable. Scattering due to the particles mean free path can be neglected. In the sputtering process, pressure influences the mean free path of sputtered atoms. A low pressure (e.g. $0.11 \mathrm{~Pa})$, sputtered particles flux is narrow compared to those obtained for high pressure (e.g. $0.53 \mathrm{~Pa}$ ). As a result, in sputtering process, the column 
DC electrical conductivity of oriented chromium thin films versus column angle $\beta$ was calculated from our proposed model and compared to measurements performed at $300 \mathrm{~K}$ with a Van Der Pauw configuration (Fig. 5). In spite of significant changes of the films' microstructure, all chromium thin films exhibit a positive Temperature Coefficient of Resistance - TCR (not shown here). TCR of the films are lower than $1.0 \times 10^{-3} \mathrm{~K}^{-1}$ (TCR of bulk chromium is $3.3 \times 10^{-3} \mathrm{~K}^{-1}$ [23]) with no clear evolution as a function of either the column angle $\beta$ or the sputtering pressure. However, for films deposited with an incident angle $\alpha=0^{\circ}$ and a low sputtering pressure $(0.11 \mathrm{~Pa}), \rho$ as well as TCR values tend to that of the bulk material. On the other hand, conductivity is particularly influenced by the column angle $\beta$ and the sputtering pressure $\mathrm{P}_{\mathrm{Ar}}$. For all argon sputtering pressures and for films deposited at normal incidence $\left(\alpha=\beta=0^{\circ}\right), \sigma_{300 \mathrm{~K}}=7.66 \times 10^{6}, 1.37 \times 10^{6}$ and $1.08 \times 10^{6} \mathrm{~S} \mathrm{~m}^{-1}$ for $\mathrm{P}_{\mathrm{Ar}}=0.11$, 0.40 and $0.53 \mathrm{~Pa}$, respectively (corresponding resistivity ratios are $\rho / \rho_{0}=1.01,5.6$ and 7.2 at $\alpha=\beta=0^{\circ}$ ). As expected, these values are lower than the conductivity of bulk chromium $\left(\sigma_{300 \mathrm{~K}}=7.74 \times 10^{6} \mathrm{~S} \mathrm{~m}^{-1}[24]\right)$. This is commonly observed in polycrystalline thin films and is directly related to the electron mean free path. This latter is strongly influenced by the scattering effects at the grain boundaries and due to the surface/interface roughness [25]. Since the conductivity exponentially decreases with respect to the number of grain boundaries per mean free path [26] and assuming an enhanced density of films sputter deposited at low pressure [27], the lowest conductivity measured for the highest sputtering pressure is mainly attributed to a decrease of microstructure defects in the films (grain boundaries, interfaces, voids, disordered atoms, ...).

For chromium thin films exhibiting an inclined columnar structure, the evolution of the resistivity ratio $\rho / \rho_{0}$ versus the column angle $\beta$ can be separated in two parts. The first one 
corresponds to a nearly constant or a slight increase of the $\rho / \rho_{0}$ ratio versus $\beta$ up to a critical value. Afterwards, ratio gains more than one order of magnitude for all sputtering pressures when the column angle $\beta$ reaches its saturated angle: $\beta=25^{\circ}$ for $0.53 \mathrm{~Pa}$ whereas $\beta=35^{\circ}$ for $0.40 \mathrm{~Pa}$ (Fig. 4). However, no saturation of $\beta$ is observed for the lowest sputtering pressure $(0.11 \mathrm{~Pa})$ and $\beta$ versus $\alpha$ well agrees with the Tait's model. It is also worth of noting that the resistivity ratio $\rho / \rho_{0}$ versus $\beta$ also depends on the sputtering pressure used to deposit chromium thin films. This critical column angle $\beta$ is again $25^{\circ}$ for $\mathrm{P}_{\mathrm{Ar}}=0.53 \mathrm{~Pa}$ whereas it is close to $35^{\circ}$ for $\mathrm{P}_{\mathrm{Ar}}=0.40 \mathrm{~Pa}$. A smooth and rapid increase of $\rho / \rho_{0}$ versus column angle $\beta$ is rather observed for films deposited at $0.11 \mathrm{~Pa}$. In addition, for a given column angle (e.g. $\beta=$ $\left.20^{\circ}\right), \sigma_{300 \mathrm{~K}} \approx 6.0 \times 10^{5} \mathrm{~S} \mathrm{~m}^{-1}\left(\rho_{0} / \rho=13\right)$ at $\mathrm{P}_{\mathrm{Ar}}=0.53 \mathrm{~Pa}$ whereas it remains higher than $1.20 \times 10^{6} \mathrm{~S} \mathrm{~m}^{-1}\left(\rho / \rho_{0}=5\right)$ at $\mathrm{P}_{\mathrm{Ar}}=0.40 \mathrm{~Pa}$ and close to $3.9 \times 10^{6} \mathrm{~S} \mathrm{~m}^{-1}\left(\rho / \rho_{0}=2\right)$ at $\mathrm{P}_{\mathrm{Ar}}=0.11$ Pa. Such a significant difference of conductivity can not be assigned to the column angle since it is the same. Modifications of the packing of the columns induced by the change of the sputtering pressure can not be neglected [27]. Thus, a low sputtering pressure favours a directional flux of the sputtered particles. As previously shown by Mahieu et al. [28], sputtered atoms incoming from the target and impinging on the growing film can at first, be assumed as a Gaussian distribution. One can suppose that more than $90 \%$ of sputtered atoms follow the incident angle $\alpha$ set by the experimental set-up. This ratio certainly reduces down to few tens percent as the argon pressure increases and the distribution of sputtered particles is spread. As a result, scattering phenomenon of the sputtered particles due to argon pressure leads to an effective incident angle. In addition and as pointed out by Fujiwara et al. [22], the main parameter that controls the columnar formation and angle $\beta$ is the preferred movement of adatoms due to their momentum and the isotropic surface diffusion. As the sputtering pressure increases, the adsorption of argon gaseous species on the film's surface is favoured. Thus, the preferred movement of adatoms prevails on isotropic surface diffusion [29, 30]. 
Columnar axis deviates from the vapour beam direction towards the films normal and column angle saturates in spite of the incident angle $\alpha$ tends to $90^{\circ}$. Therefore, strategy about the choice of the appropriate argon pressure has to be though when depositing oriented columnar structure by sputtering. In order to calculate the electrical resisitivity ratio $\rho / \rho_{0}$ versus column angle $\beta$ with equation (25), the evolution of the film's density $\xi$ as a function of the incident angle $\alpha$ was computed according to equation (7) and taking into account the effect of the sputtering pressure (Table 1). The film's density decreases monotonically with increasing $\alpha$. The constant $\mathrm{c}$ (proportional to the ratio of the shadowing step height to the column length [16]) and bulk density $\xi_{0}$ have empirically been determined in agreement with earlier simulations [16, 31, 32] and experimental results [9, 33-35].

The significant rising of resistivity ratio $\rho / \rho_{0}$ measured for a given $\beta$ angle is in good agreement with previous investigations [36] and an interesting recent study focused on conduction anisotropy in porous thin films. Vick and Brett [37] also observed a rapid decline in the conductivity, accompanied by an increase of conduction anisotropy in the plane of the substrate as a function of the incident angle $\alpha$ and consequently, the column angle $\beta$. In our study, the Van Der Pauw geometry was implemented. It does not allow probing of the conduction anisotropy without some specific configuration of the contacts [38] since the method is based on reciprocal measurements. As a result, conduction anisotropy can not be brought to the fore with a conventional method. An averaged measurement is rather performed but clearly exhibits a strong dependence with the microstructure and orientation of the columns. It is also confirmed from the theoretical conductivity versus column angle $\beta$ calculated from our proposed model (Fig. 5). A good agreement is observed between measured and simulated data. The drop of electrical conductivity is well predicted for all sputtering pressures. Critical column angles $\beta$ close to $25^{\circ}$ for $\mathrm{P}_{\mathrm{Ar}}=0.53 \mathrm{~Pa}$ and $35^{\circ}$ for $\mathrm{P}_{\mathrm{Ar}}=$ 0.40 $\mathrm{Pa}$ deduced from observations of the cross-section of the films, are also expected by the 
proposed model. It is also worth of noticing that the smooth and gradual increase of the calculated resistivity ratio $\rho / \rho_{0}$ versus column angle $\beta$ exhibits a very good agreement with experimental measurements, which supports again the model. Consistency and agreement between calculated and measured conductivity versus column angle $\beta$ validates the pertinence of our model. In addition, the fall of the observed conductivity for a critical column angle is well predicted by the theory and taking into account the sputtering pressures. It also corroborates that this model can be used to anticipate the role of some sputtering process parameters on the transport properties of metallic films produced by GLAD. This significant increase of the electrical resistivity ratio measured for column angles $\beta$ close to $55^{\circ}$ for $\mathrm{P}_{\mathrm{Ar}}=$ $0.53 \mathrm{~Pa}$ against $30^{\circ}$ for $\mathrm{P}_{\mathrm{Ar}}=0.40 \mathrm{~Pa}$, can be related to some modifications of the films' microstructure. Since no change of the preferred orientation versus column angle was observed (not presented here) and due to a linear increase of the crystallite size (by XRD) from 25 to $45 \mathrm{~nm}$ as the incident angle $\alpha$ rises from 0 to $85^{\circ}$, the contribution of disorder and/or organization at the atomic range can not be taken into account to explain the increase of the electrical resistivity in these oriented chromium thin films. One should expect an enhanced rather than a drop of conductivity since crystallites become bigger. On the other hand, an enhancement of a more porous structure is quite favoured as the incident angle $\alpha$ rises [39]. Previous studies devoted to GLAD thin films clearly demonstrated an increase of the porosity (density of the films less than $30 \%$ of the bulk density [12]), especially for incident angles higher than $70^{\circ}$ [40]. It well agrees with our calculated film's density versus $\alpha$ obtained from Paik's law [16] (Table 1). As a result, this conductivity decline has to be assigned to the enhancement of the electron scattering by surfaces and grain boundaries induced by a much more porous structure promoted as the incident angle $\alpha$ tends to $90^{\circ}$.

It is worth of noting some slight discrepancies between theoretical and experimental values. Due to the good agreement between modelled and measured resistivity ratios, 
improvements proposed below appear as second order considerations. At first, one can suggest that these discrepancies have to be attributed to accuracy of the conductivity measurements, but also to some starting hypotheses we supposed on the columnar structure in order to simplify the calculations. Beside some bases of the model are restrictive, one can bring some improvements on the description of the structure in order to better predict the electrical properties. Since oriented columns are produced, anisotropy of the structure should be considered [37, 41, 42]. In our proposed model, it is implicitly taken into account since the column angle, the width as well as the length of the columns and especially the direction of the column growth are considered in the calculations. However, our assumptions considered squared columnar sections and an isotropic medium in $\mathrm{x}$ and $\mathrm{y}$ directions in order to simplify calculations. From Tait et al. [36], it has been shown that columns tend to exhibit sections with an elongated shape in the direction perpendicular to the plane of incidence of the particles flux (elliptical column shapes) [43]. This phenomenon was neglected and could be integrated in our model (column width $\mathrm{D}_{\mathrm{x}}$ differs to $\mathrm{D}_{\mathrm{y}}$ ) so as to consider anisotropic effects due to shadowing effect during thin film growth. In addition, section of the columns is assumed as a constant through the length $D_{z}$ (i.e. $D_{x}$ and $D_{y}$ increases versus thickness of the film). Vanishing of some columns due to shadowing effect is also neglected. It may contribute significantly to the transport properties since electrical conduction strongly depends on the nature and the quality of the film/substrate interface [44].

Another improvement of the model can be proposed since the vacuum deposition of thin films exhibiting a columnar structure commonly induces perturbations of the surface/interface roughness [25, 45]. It is often more justified for coatings with an oriented columnar architecture. Contribution of the grain boundaries on the electronic scattering is frequently assumed in numerous models focused on the electrical conductivity of thin films [46-52]. Nevertheless, roughness effects have to be encountered similarly. Such effects can 
not be omitted whenever the roughness correlation length is comparable or larger than the grain size [25]. Therefore, further investigations in this direction is required in order to also describe surface/interface scattering mechanisms on transport properties of polycrystalline and oriented thin films.

Last but not least, our approach could be even more realistic for metallic thin films assuming the oxidation of the films that certainly occurs as the structure becomes more and more porous. Vick and Brett [37] also suggested the oxidation effect of metallic surfaces as a possible source of errors between theoretical and experimental conductivity in titanium GLAD thin films. Strong reactivity of oxygen toward metals is especially important for elements like $\mathrm{Ti}, \mathrm{Nb}, \mathrm{Zr}, \ldots$ Native oxide layer of few nanometres thickness is formed spontaneously on exposed metallic surfaces. As a result, oxidation of the samples is an unavoidable consequence after the film deposition. The impact of such layer becomes significant for high column angles (i.e. as the porous structure is favoured). Thus, oxidation kinetics have to be taken into account since they depend on the nature of sputtered compound (reactivity of the metallic surfaces toward oxygen), temperature versus conductivity measurements (temperature coefficient of resistance may also be affected [25]) and architecture of the films.

\section{Conclusions}

A simple theoretical model has been proposed to calculate the DC electrical conductivity of metallic thin films exhibiting an inclined columnar structure. The theory presented in this paper has been shown to give good predictions of the films' conductivity as a function of the column angle $\beta$. Structural characteristics of the films like column angle, grain and column sizes as well as some process parameters like the sputtering pressure have been taken into account in the development of the analytical model. For a given column angle 
(critical angle), an abrupt decrease of the conductivity has been computed. Such a critical angle depends on the sputtering pressure involved to deposit the films.

In order to validate the theoretical model, DC electrical conductivity has been measured using the Van Der Pauw configuration for chromium thin films sputter deposited using the GLAD technique. Although some restrictive hypotheses assumed as a starting point in the theoretical approach and in spite of an averaging effect peculiar to the four probes method (anisotropic effect is neglected at first), a good agreement has been noticed between calculated and experimental electrical properties. A systematic change of the incident angle $\alpha$ of the particles flux has been performed in order to deposit various oriented columnar structures and using different sputtering pressures. Conductivity measurements also exhibited a critical column angle from which conductivity has been reduced. The predicted shift of the critical angle to higher values has also been observed for experiments as the sputtering pressure increased.

Finally, the presented model is suitable to foresee the evolution of electrical conductivity in oriented metallic films. Geometry and dimensions of the columnar architecture have to be considered similarly to the process parameters so as to predict the transport properties in sculptured thin films produced by GLAD sputtering.

\section{Acknowledgements}

The authors thank C. Millot for SEM observations and G. Michel for technical helps. The city of Besançon is especially acknowledged for financial support. 


\section{References}

[1] Seto J Y W 1975 J. Appl. Phys. 46(12) 5247

[2] Ueda Y, Takakura W and Yamada A 2002 J. Magn. Mater. 239457

[3] Knäbchen A 1995 Thin Solid Films 265107

[4] Tellier C R and Tosser A J 1976 Electrocomponent Sci. Technol. 3165

[5] Du H, Chen H, Gong J, Wang T G, Sun C, Lee S W and Wen L S 2004 Appl. Surf. Sci. 23399

[6] J.R. Sambles J R 1983 Thin Solid Films 106321

[7] E.E. Mola E E and J.M. Heras J M, 1974 Electrocomponent Sci. Technol. 177

[8] Baccarani G, Ricco B and Spadini G 1978 J. Appl. Phys. 49(11) 5565

[9] Pandya D K, Rastogi A C and Chopra K L 1975 J. Appl. Phys. 46(7) 2966

[10] Steeves M M, Deniz D and Lad R J 2010 Appl. Phys. Lett. 96142103

[11] Lintymer J, Martin N, Chappé J M, Delobelle P and Takadoum J 2004 Surf. Coat. Technol. 180-181 26

[12] Robbie K, Sit J C and Brett M J 1998 J. Vac. Sci. Technol. B16(3) 1115

[13] Thornton J A 1974 J. Vac. Sci. Technol. 11666

[14] Mayadas A F and Shatzkes M 1970 Phys. Rev. B 11382

[15] Pichard C R, Tellier C R and Tosser A J 1979 Thin Solid Films 62189

[16] Paik S M, Kim S, Schuller I K and Ramirez R 1991 Phys. Rev. B 43(2) 1843

[17] Cottey A A 1968 Thin Solid Films 4297

[18] Van Der Pauw L J 1958 Philips Tech. Rev. 20220

[19] Nieuwenhuizen J M and Haanstra H B 1966 Philips Tech. Rev. 2787

[20] Tait R N, Smy T and Brett M J 1993 Thin Solid Films 226196

[21] Yamamura Y and Muraoka K 1989 Nucl. Intrum. Methods B42 175 
22] Fujiwara H, Hara K, Kamiya M, Hashimoto T and Okamoto K 1998 Thin Solid Films 163387

[23] Cook J G 1985 Thin Solid Films 129 L57

[24] Kulkarni A K and Chang L C 1997 Thin Solid Films 30117

[25] Palasantzas G 1998 Phys. Rev. B 58(15) 9685

[26] Reiss R, Vancea J and Hoffmann H 1986 Phys. Rev. Lett. 56(19) 2100

[27] Itoh K, Kamiya M, Hara K, Hashimoto T, Okamoto K and Fujiwara H 1991 Thin Solid Films 185245

[28] Mahieu S, Buyle G, Depla D, Heirwegh S, Ghekiere P and De Gryse R 2006 Nucl. Intrum. Methods B243 313

[29] Fujiwara H 1988 Thin Solid Films 163379

[30] Hashimoto T, Hara K, Okamoto K and Fujiwara H 1976 J. Phys. Soc. Jpn. 411433

[31] Leamy H J and Dirks A G 1977 J. Phys. D 10 L95

[32] Muller A H 1985 J. Appl. Phys. 582573

[33] Buzea C and Robbie K 2004 J. Optoelectronics Advanced Mater. 61263

[34] Nadhodkin N G and Shaldervan A I 1972 Thin Solid Films 10109

[35] Buzea C, Kaminska K, Beydaghyan G, Brown I, Elliott C, Dean C and Robbie K 2005 J. Vac. Sci. Technol. B23 2545

[36] Tait R N, Dew S K, Smy T and Brett M J 1991 J. Appl. Phys. 70(8) 4295

[37] Vick D and Brett M J 2006 J. Vac. Sci. Technol. A24(1) 156

[38] Kleiza J, Sapagovas M and Kleiza V 2007 Informatica 18(2) 253

[39] Asgharizadeh S, Sutton M, Robbie K and Brown T 2009 Phys. Rev. B 79125405

[40] Smy T, Vick D, Brett M J, Dew S K, Sit J C and Harris K D 2000 J. Vac. Sci. Technol. A18(5) 2507

[41] Kuwara K and Shinzato S 1988 Thin Solid Films 164165 
[42] Kuwara K and Hirota H 1974 Jpn. J. Appl. Phys. 131093

[43] Tait R N, Smy T and Brett M J 1992 J. Vac. Sci. Technol. A10(4) 1518

[44] Sambles J R 1983 Thin Solid Films 106321

[45] Au Yeung T C, Chiam T C, Chen C K, Sun C Q, Shangguan W Z, Wong W K and Kam C H 2005 Phys. Rev. B 72155417

[46] Knight G D and Smy T 2002 Microelectron. Eng. 64417

[47] Zhang W, Brongersma S H, Richard O, Brijs B, Palmans R, Froyen L and Maex K 2002 Microelectron. Eng. 64417

[48] Marom H, Ritterband M and Eizenberg M 2006 Thin Solid Films 51062

[49] Nan C W, Tschöpe A, Holten S, Kliem H and Birringer R 1999 J. Appl. Phys. 85(11) 7735

[50] Seager C H and Pike G E 1982 Appl. Phys. Lett. 40(6) 471

[51] Muller R K 1961 J. Appl. Phys. 32(4) 635

[52] Fuertes Marrin D, Sadewasser S, Meeder A, Glatzel T and Lux-Steiner M C 2005 Phys. Rev. B 7133306 


\section{Table caption}

\section{Table 1}

Bulk density $\xi_{0}$ and constant c used to calculate the theoretical film's density $\xi$ (\% of the bulk material's density) as a function of the incident angle $\alpha$ for three sputtering pressures according to equation (7) proposed by Paik et al. [16]. 
Table 1

\begin{tabular}{ccc}
\hline Pressure (Pa) & Density of the film $\boldsymbol{\xi}_{\mathbf{0}}$ for $\boldsymbol{\alpha}=\mathbf{0}^{\circ}$ & Constant c \\
\hline 0.11 & 100 & 0.06 \\
0.40 & 98 & 0.02 \\
0.53 & 95 & 0.015 \\
\hline
\end{tabular}




\section{Figure captions}

Figure 1

Geometric definition of distances $\mathrm{L}_{\mathrm{x}}, \mathrm{L}_{\mathrm{y}}, \mathrm{L}_{\mathrm{z}}$ and interplanar spacings $\mathrm{D}_{\mathrm{x}}, \mathrm{D}_{\mathrm{y}}, \mathrm{D}_{\mathrm{z}}$; a) Along the Ox direction; b) Along the Oy direction; c) Along the Oz direction.

\section{Figure 2}

Schematic draw of an inclined (squared section) column; $\beta=$ angle of the column; $d=$ height of the column; $\mathrm{D}_{\mathrm{x}}=$ width of the column.

\section{Figure 3}

Cross-section observations by SEM of chromium thin films sputter deposited on (100) Si with a) $\alpha=85^{\circ}\left(\beta=21^{\circ}\right)$ and $\mathrm{P}_{\mathrm{Ar}}=0.53 \mathrm{~Pa}$; b) $\alpha=85^{\circ}\left(\beta=38^{\circ}\right)$ and $\mathrm{P}_{\mathrm{Ar}}=0.40 \mathrm{~Pa}$; $) \alpha=85^{\circ}(\beta$ $\left.=60^{\circ}\right)$ and $\mathrm{P}_{\mathrm{Ar}}=0.11 \mathrm{~Pa}$.

\section{Figure 4}

Evolution of the column angle $\beta$ versus incident angle $\alpha$ in chromium thin films deposited by sputtering for three different argon pressures. Comparison with empirical tangent rules and Tait's relationships between $\alpha$ and $\beta$ angles. Saturation of the column angle $\beta$ is measured as incident angle $\alpha$ tends to $90^{\circ}$ for high sputtering pressures $(0.40$ and $0.53 \mathrm{~Pa})$.

\section{Figure 5}

Electrical resistivity ratio $\rho / \rho_{0}$ of chromium thin films measured at $300 \mathrm{~K}$ and calculated from our proposed model as a function of the column angle $\beta$. The effect of the argon sputtering pressure on the $\rho / \rho_{0}$ ratio is also illustrated. 
Figure 1 a)

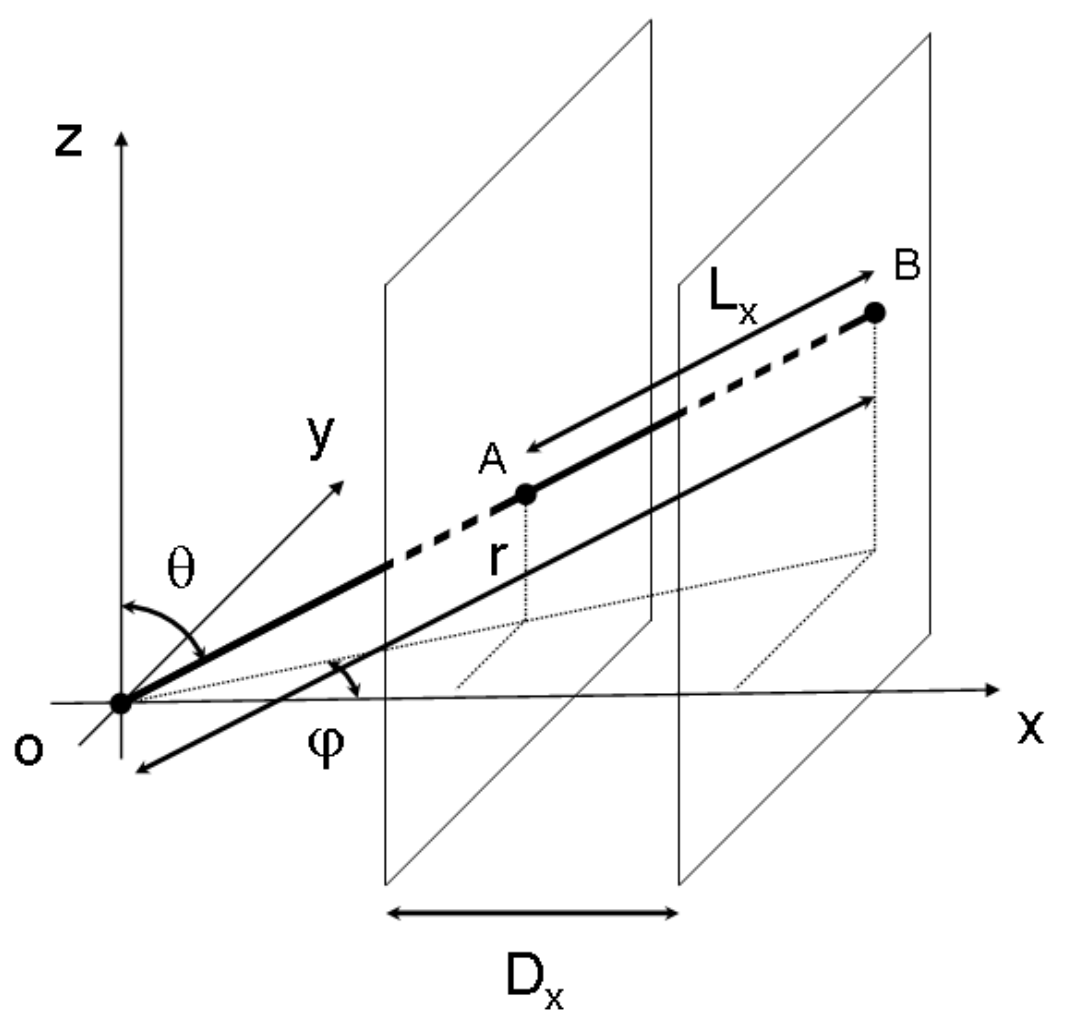

Besnard et al.

Figure 1a (Figure1a.tif) 
Figure $1 b$ )

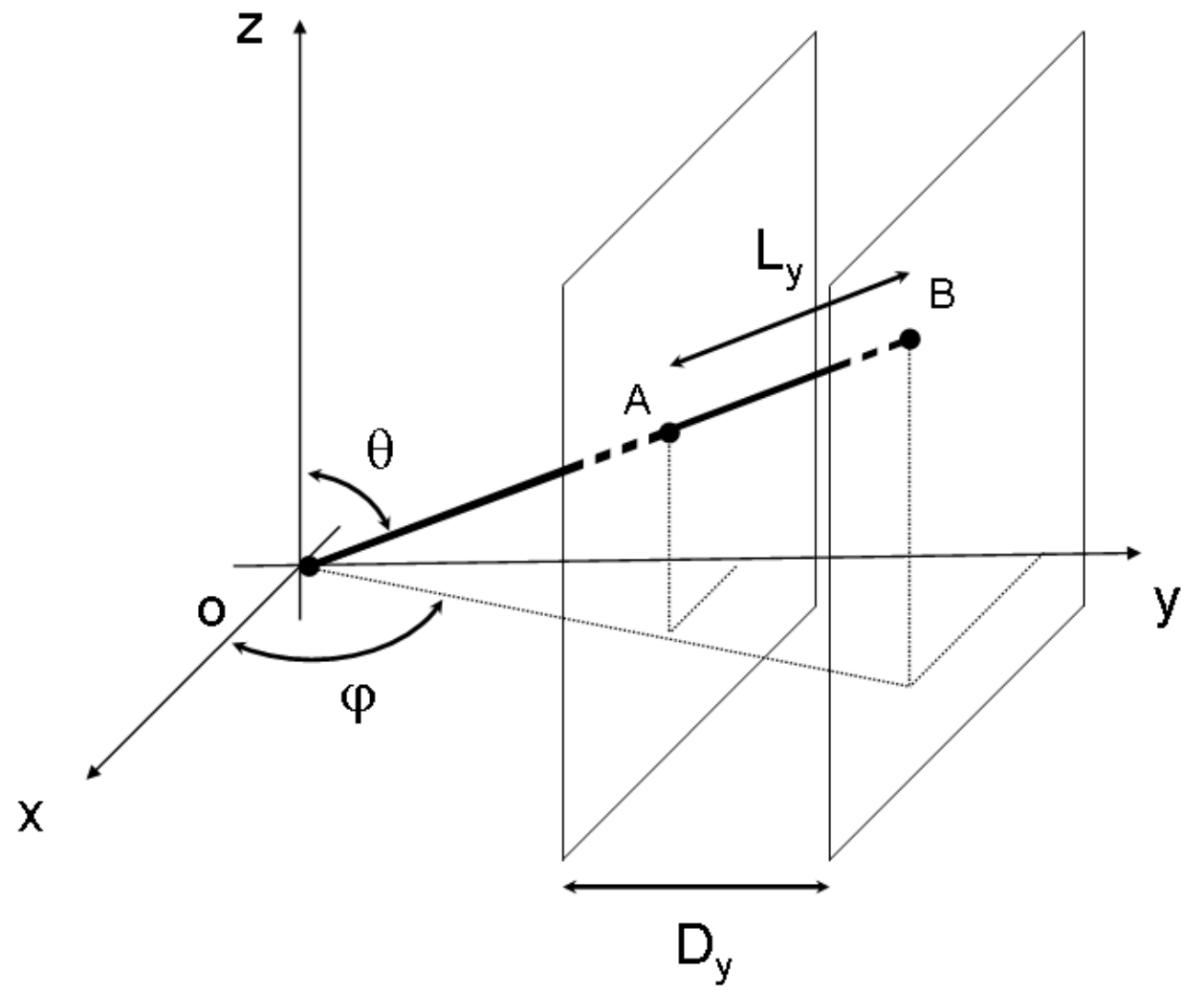

Besnard et al.

Figure 1b (Figure1b.tif) 
Figure 1 c)

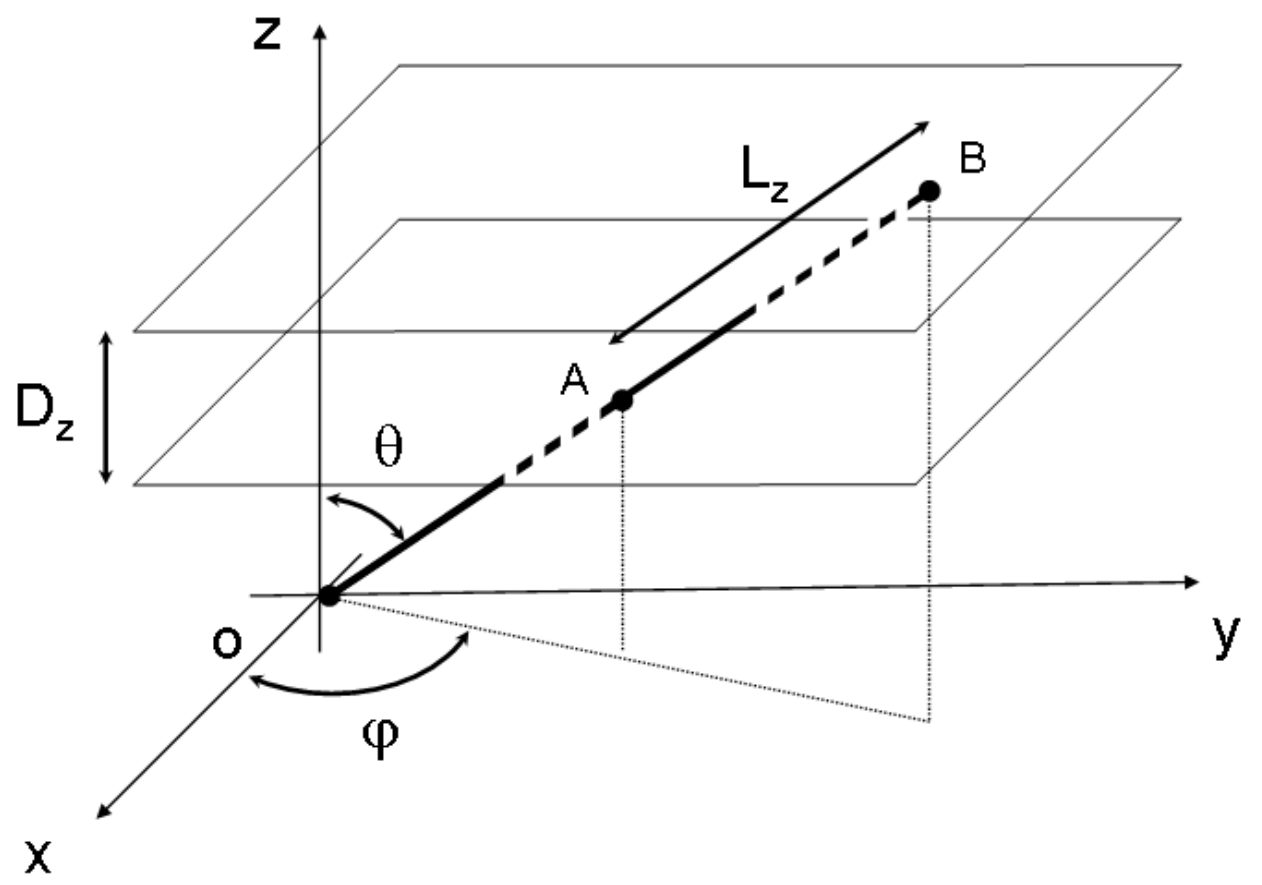

Besnard et al.

Figure 1c (Figure1c.tif) 
Figure 2

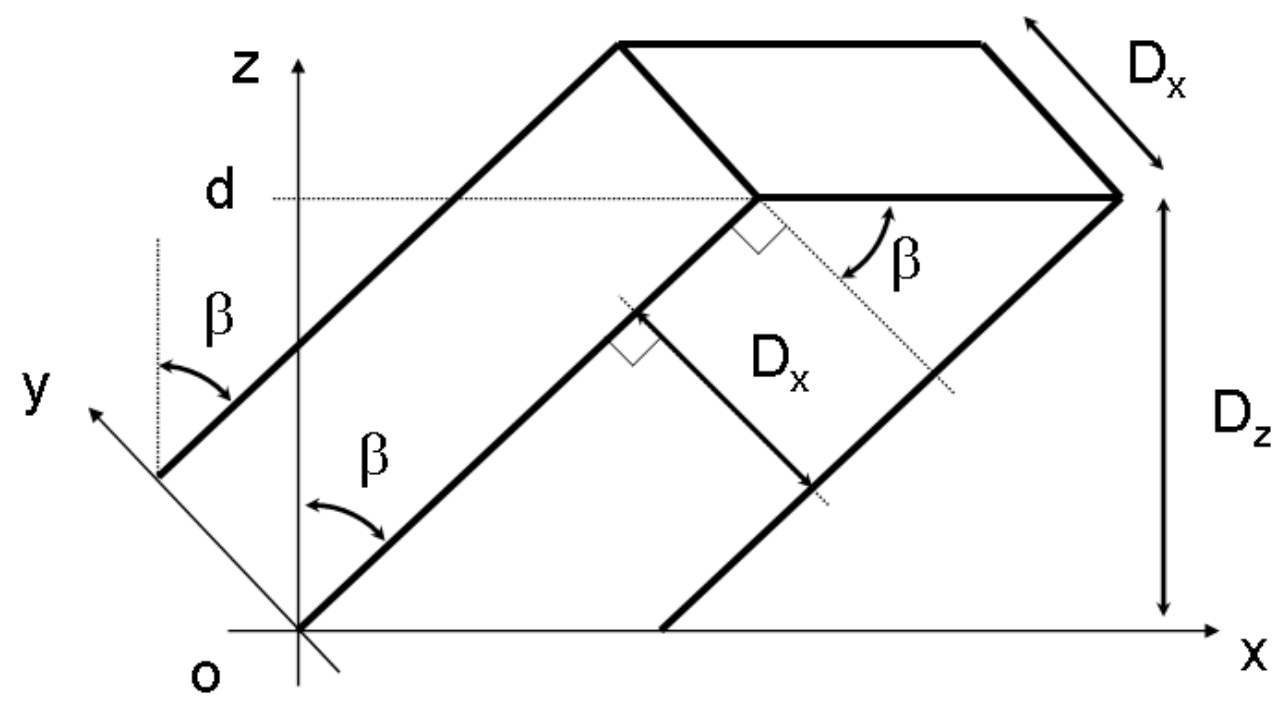

Besnard et al.

Figure 2 (Figure2.tif) 
Figure 3 a)

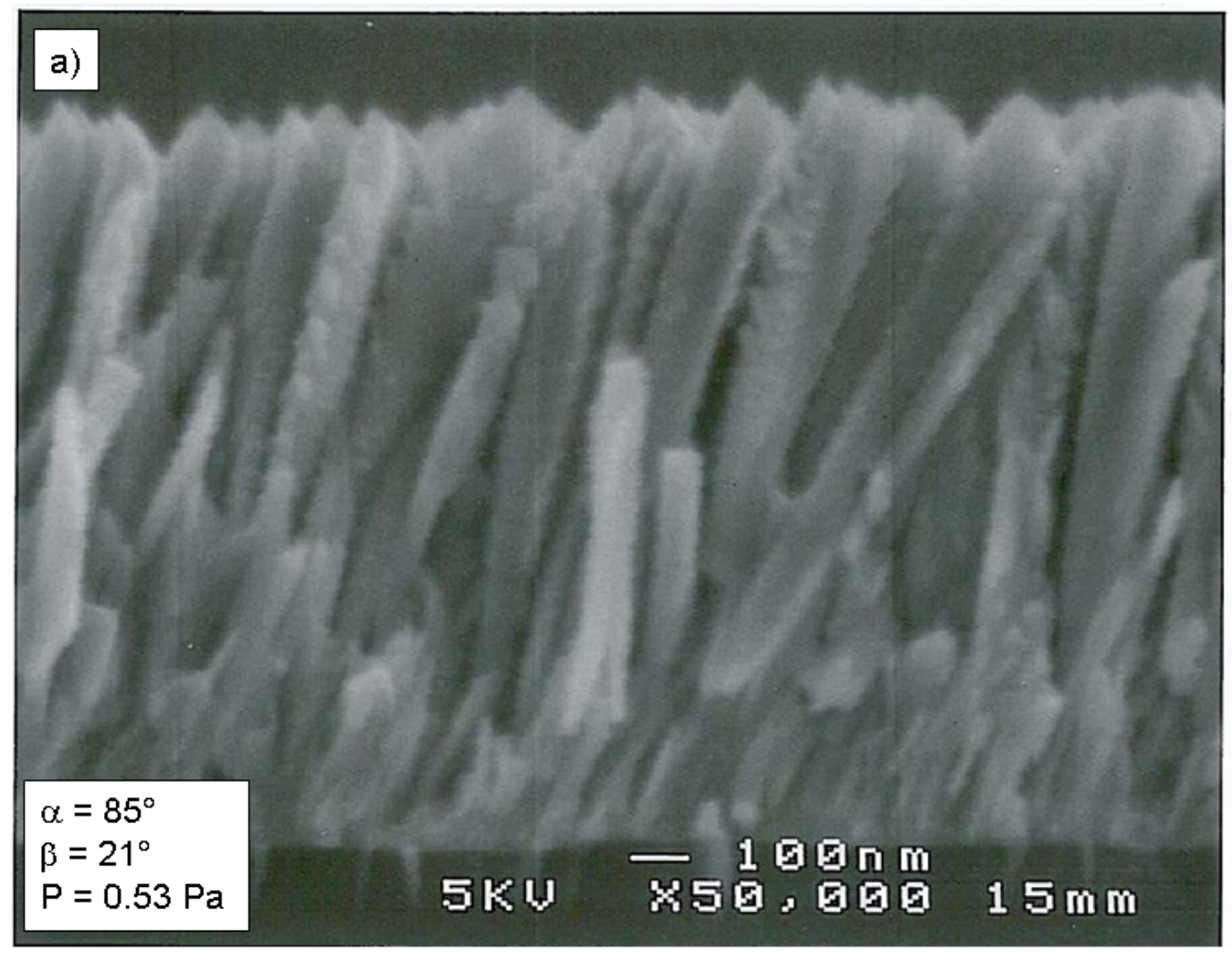

Besnard et al.

Figure 3a (Figure3a.tif) 
Figure $3 b$ )

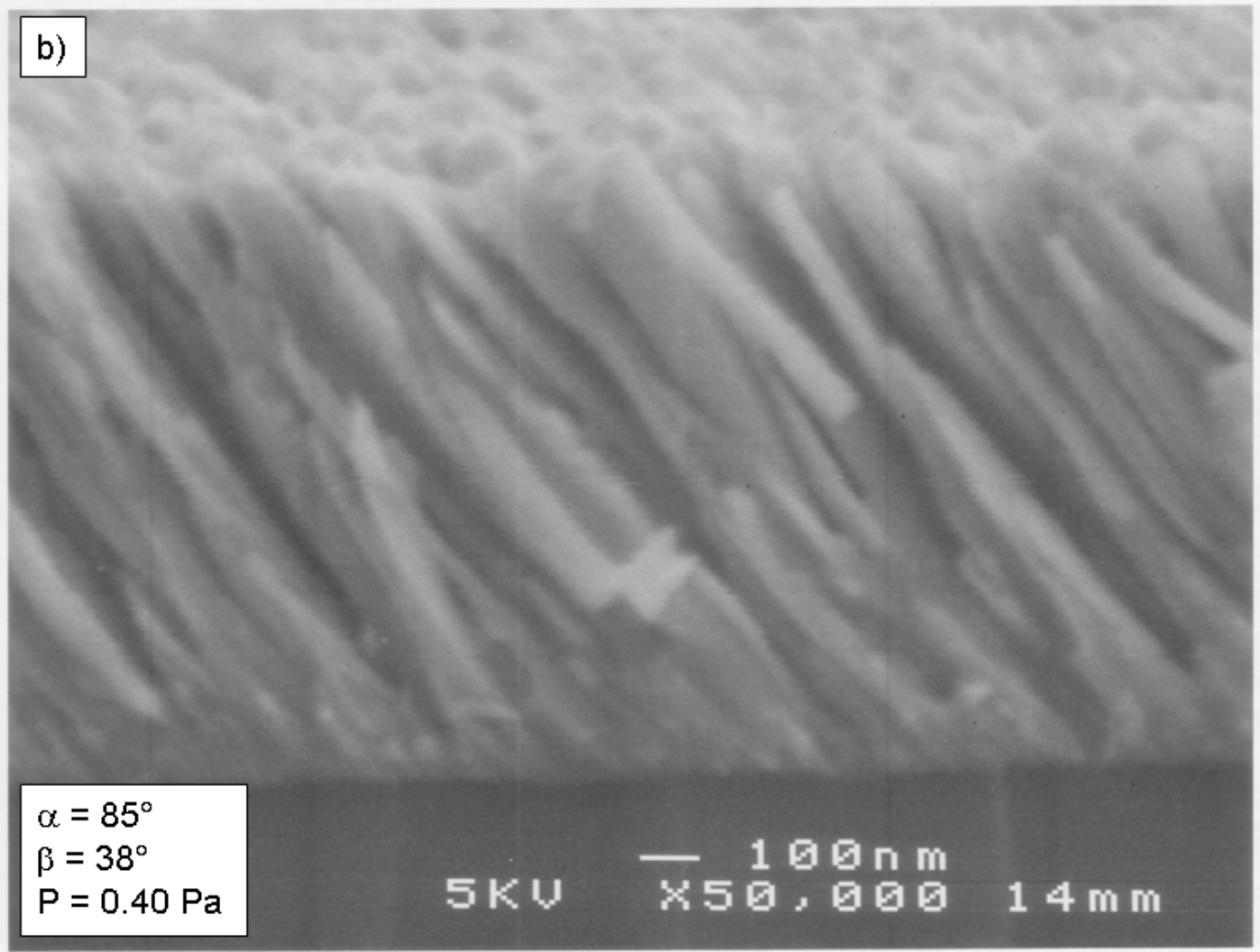

Besnard et al.

Figure 3b (Figure3b.tif) 
Figure $3 \mathrm{c}$ )

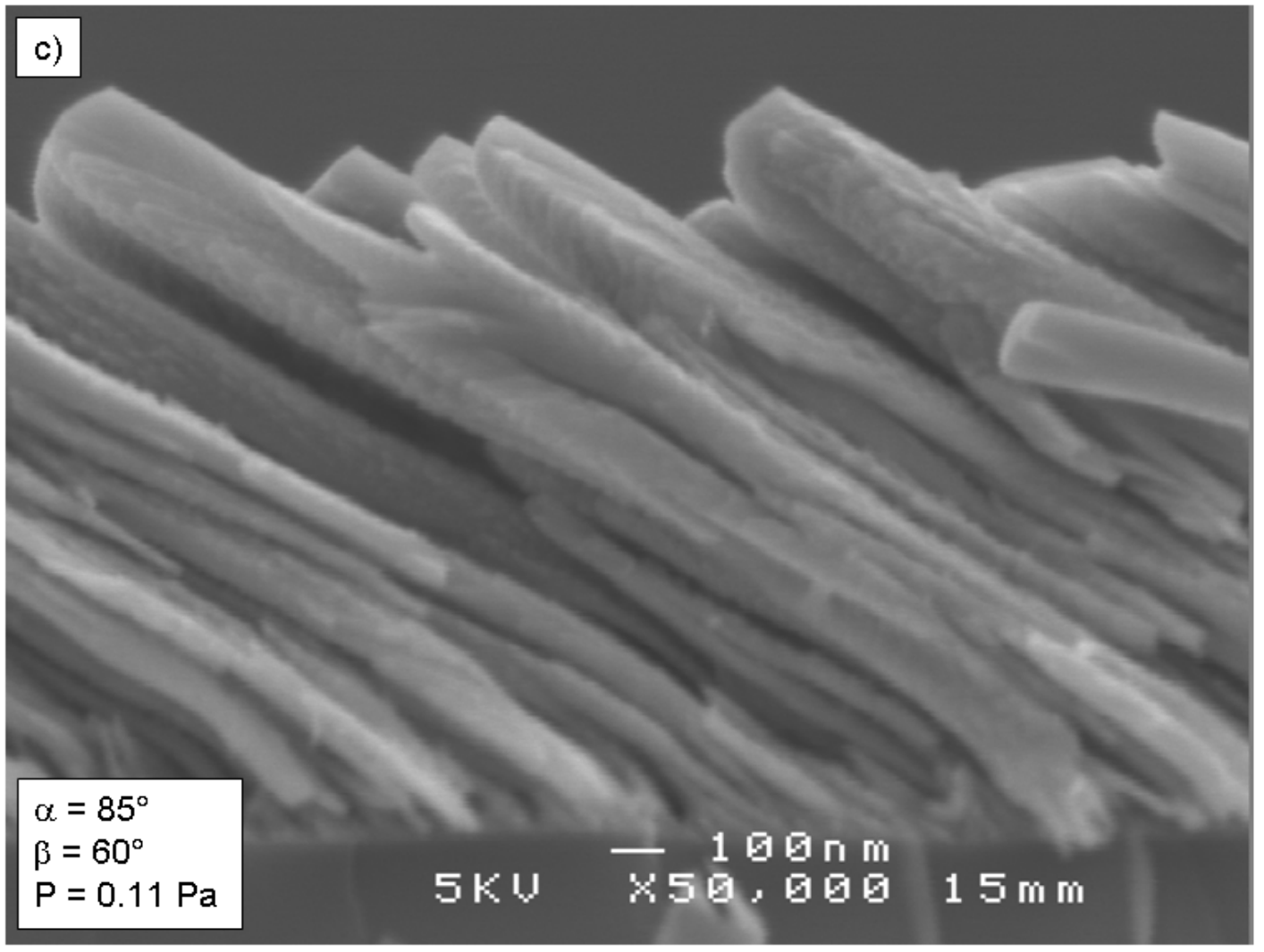

Besnard et al.

Figure 3c (Figure3c.tif) 
Figure 4

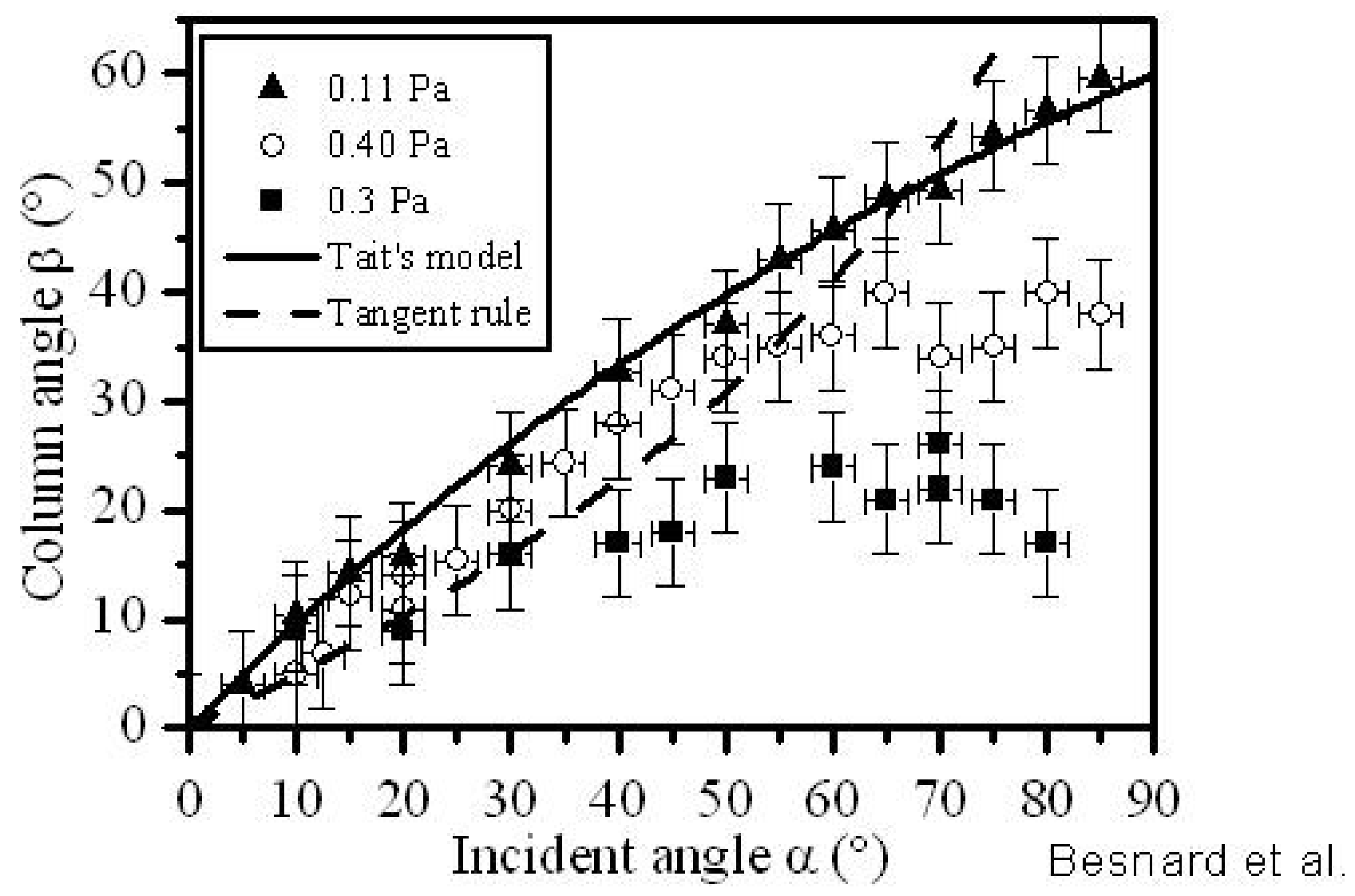

Figure 4 (Figure4.tif) 
Figure 5

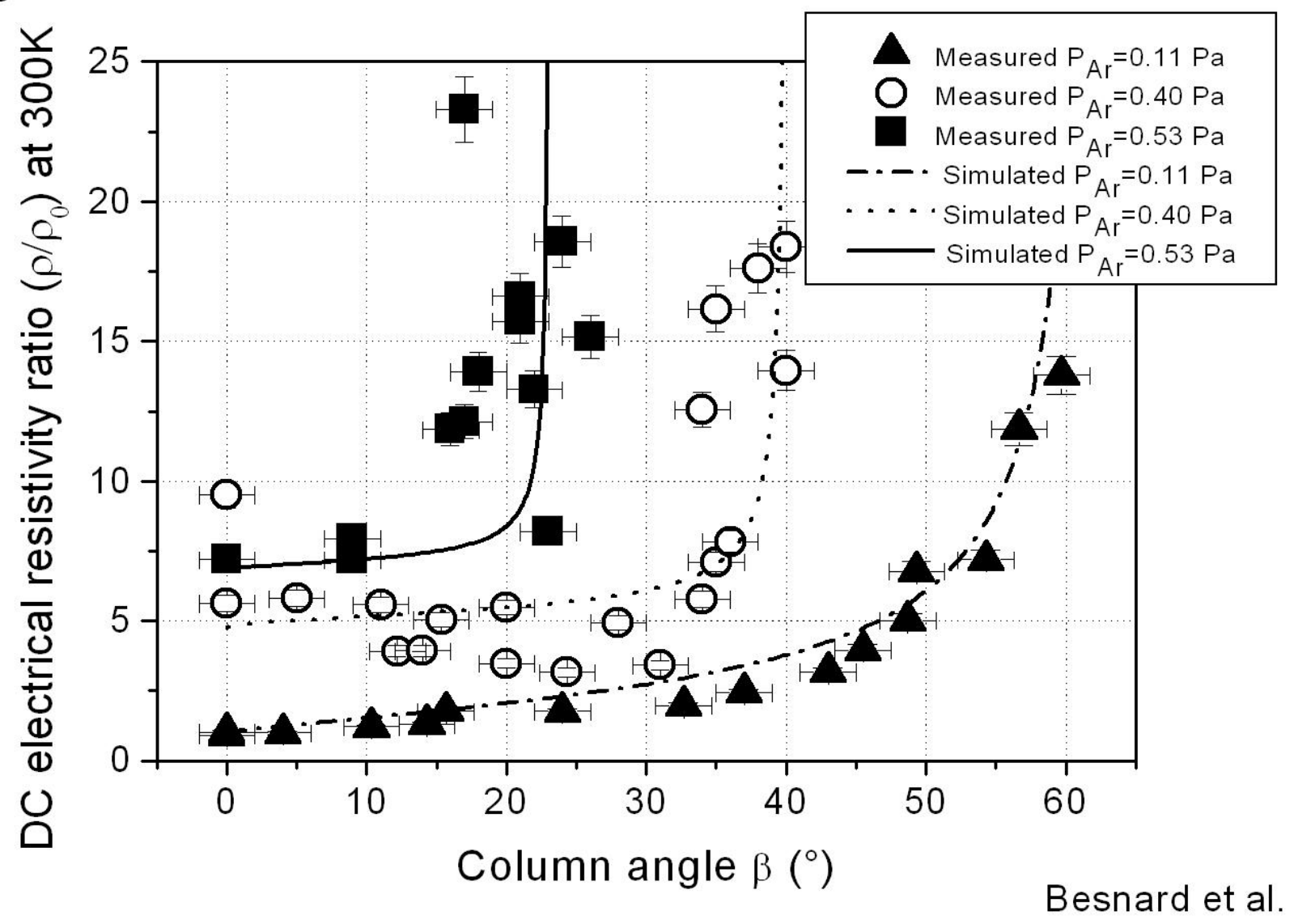

Figure 5 (Figure5.tif) 